\title{
Hanbury Brown-Twiss Interferometry at a Free-Electron Laser
}

\author{
A. Singer, ${ }^{1}$ U. Lorenz, ${ }^{1}$ F. Sorgenfrei, ${ }^{2, *}$ N. Gerasimova, ${ }^{1}$ J. Gulden, ${ }^{1, \dagger}$ O. M. Yefanov, ${ }^{1, \ddagger}$ R. P. Kurta, ${ }^{1}$ A. Shabalin, ${ }^{1}$ \\ R. Dronyak, ${ }^{1}$ R. Treusch, ${ }^{1}$ V. Kocharyan, ${ }^{1}$ E. Weckert, ${ }^{1}$ W. Wurth,,${ }^{2, \S}$ and I. A. Vartanyants ${ }^{1,3, \|}$ \\ ${ }^{1}$ Deutsches Elektronen-Synchrotron DESY, Notkestrasse 85, D-22607 Hamburg, Germany \\ ${ }^{2}$ Institut für Experimentalphysik and CFEL, University of Hamburg, Luruper Chaussee 149, D-22603 Hamburg, Germany \\ ${ }^{3}$ National Research Nuclear University, "MEPhI", 115409 Moscow, Russia
}

(Received 28 January 2013; published 17 July 2013)

\begin{abstract}
We present measurements of second- and higher-order intensity correlation functions (so-called Hanbury Brown-Twiss experiment) performed at the free-electron laser (FEL) FLASH in the non-linear regime of its operation. We demonstrate the high transverse coherence properties of the FEL beam with a degree of transverse coherence of about $80 \%$ and degeneracy parameter of the order $10^{9}$ that makes it similar to laser sources. Intensity correlation measurements in spatial and frequency domain gave an estimate of the FEL average pulse duration of $50 \mathrm{fs}$. Our measurements of the higher-order correlation functions indicate that FEL radiation obeys Gaussian statistics, which is characteristic to chaotic sources.
\end{abstract}

Hanbury Brown and Twiss, in their pioneering experiments $[1,2]$, demonstrated that one can get fundamental information on the statistics of light sources by measuring intensity correlations at two separated spatial positions. Originally designed as a robust method to determine the size of stars, these experiments initiated developments in the field of quantum optics [3]. Statistical properties of thermal sources [1,2], lasers [4], semiconductor microcavities [5], and, recently, Bose-Einstein condensates [6,7] have been studied using this technique. The recent advent of x-ray free-electron lasers (FELs) [8-11] with their unprecedented peak brilliance and ultrashort pulse duration has opened the route to a number of spectacular groundbreaking experiments including femtosecond nanocrystallography [12] and single particle coherent imaging [13,14]. Many of these experiments exploit the high degree of coherence of the FELs.

Coherence is the defining feature of a laser source and is described by correlation functions within statistical optics $[15,16]$. The first-order correlation properties of FEL sources have been extensively investigated recently [17-22]. It was experimentally demonstrated that FELs based on the self-amplified spontaneous emission (SASE) process have a high degree of transverse coherence but poor temporal coherence. To get a more detailed picture of the statistical properties of these sources, higher-order field correlations must be studied. These can be explored, for example, by utilizing intensity correlation measurements in a Hanbury Brown-Twiss (HBT) experiment. In this Letter we present measurements of second- and higherorder intensity correlation functions at the free-electron laser FLASH [8].

The core idea of the HBT experiment [1,2] is to determine the normalized second-order intensity correlation function

$$
g^{(2)}\left(\mathbf{r}_{1}, \mathbf{r}_{2}\right)=\frac{\left\langle I\left(\mathbf{r}_{1}\right) \cdot I\left(\mathbf{r}_{2}\right)\right\rangle}{\left\langle I\left(\mathbf{r}_{1}\right)\right\rangle\left\langle I\left(\mathbf{r}_{2}\right)\right\rangle}
$$

by measuring the coincident response of two detectors at separated positions $\mathbf{r}_{1}$ and $\mathbf{r}_{2}$ (see for review [23]). In Eq. (1), $I\left(\mathbf{r}_{1}\right), I\left(\mathbf{r}_{2}\right)$ are the intensities of the wave field, and the averaging is done over a large ensemble of different realizations of the wave field. It is well established that chaotic light can be described in the frame of Gaussian statistics [15] and is completely determined in spatial domain by the first-order correlation function known as the normalized spectral degree of coherence (SDC) $\mu\left(\mathbf{r}_{1}, \mathbf{r}_{2}\right)$. It is defined as [15] $\mu\left(\mathbf{r}_{1}, \mathbf{r}_{2}\right)=$ $W\left(\mathbf{r}_{1}, \mathbf{r}_{2}\right) / \sqrt{S\left(\mathbf{r}_{1}\right) S\left(\mathbf{r}_{2}\right)}$, where $W\left(\mathbf{r}_{1}, \mathbf{r}_{2}\right)$ is the cross spectral density function and $S(\mathbf{r})$ is the spectral density.

The intensity correlation function then reduces to [24]

$$
g^{(2)}\left(\mathbf{r}_{1}, \mathbf{r}_{2}\right)=1+\zeta_{2}\left(D_{\omega}\right)\left|\mu\left(\mathbf{r}_{1}, \mathbf{r}_{2}\right)\right|^{2},
$$

where the contrast function $\zeta_{2}\left(D_{\omega}\right)$ strongly depends on the bandwidth $D_{\omega}$ of the radiation. It was earlier demonstrated [25] that for stationary chaotic sources the contrast $\zeta_{2}\left(D_{\omega}\right)$ is determined by the ratio $\tau_{c} / T$, where $\tau_{c}=2 \pi / D_{\omega}$ is the coherence time of the wave field and $T$ is the time resolution of the detectors. Equivalently, the contrast $\zeta_{2}\left(D_{\omega}\right)$ determines the number of longitudinal modes $M_{T}=1 / \zeta_{2}\left(D_{\omega}\right)$ for a chaotic source. By definition the SDC $\left|\mu\left(\mathbf{r}_{1}, \mathbf{r}_{2}\right)\right| \leq 1$ and intensity correlation function $g^{(2)}\left(\mathbf{r}_{1}, \mathbf{r}_{2}\right) \leq 2$.

For the full statistical description of the wave field the $n$th order correlation functions can be introduced $[3,15]$

$$
g^{(n)}\left(\mathbf{r}_{1}, \ldots, \mathbf{r}_{n}\right)=\frac{\left\langle\prod_{i=1}^{n} I\left(\mathbf{r}_{i}\right)\right\rangle}{\prod_{i=1}^{n}\left\langle I\left(\mathbf{r}_{i}\right)\right\rangle} .
$$

For chaotic light $g^{(n)}\left(\mathbf{r}_{1}, \ldots, \mathbf{r}_{n}\right)$ is completely described by the SDC $\mu\left(\mathbf{r}_{1}, \mathbf{r}_{2}\right)$ due to the Gaussian moment theorem $[15,24]$. A comparison between correlation functions of different orders determines whether the field obeys Gaussian statistics or not. In particular, for chaotic light 
the $n$th order correlation function $g^{(n)}(\mathbf{r}, \mathbf{r}, \ldots, \mathbf{r})$ is equal to $n$ ! for a single longitudinal mode [15].

For pulsed sources the intensity correlation measurements are naturally gated by the pulse duration $T$ [25]. FEL sources, with pulses of few tens of femtoseconds, are ideally suited for intensity correlation measurements. According to FEL theory [26,27] these sources should obey Gaussian statistics in the linear and deep nonlinear regime of operation. In these conditions the correlation function $g^{(2)}\left(\mathbf{r}_{1}, \mathbf{r}_{2}\right)$ has the form of Eq. (2) and provides access to the transverse coherence properties of an FEL as well as to its pulse duration [25].

The experiment was carried out at FLASH that was operated with six undulator modules and a total undulator length of $30 \mathrm{~m}$. The electron bunch charge was $600 \mathrm{pC}$, and the electron energy $1.08 \mathrm{GeV}$ resulting in a photon wavelength of $\lambda=5.5 \mathrm{~nm}$. The average photon pulse energy was about $110 \mu \mathrm{J}$, which corresponds to about $3 \times 10^{12}$ photons per pulse at this photon energy. The measurements were performed at the PG2 beam line [28,29] (see Fig. 1). The optical system focuses the beam at a distance of $71.5 \mathrm{~m}$ downstream from the undulator exit. A monochromator comprised of a plane grating (PG), collimating (M1) and focusing (M2) mirrors, and an exit slit with variable slit width was utilized to modify the bandwidth. The plane grating has a line density of 200 lines $/ \mathrm{mm}$ and was tuned to its third order resulting in a dispersion in the exit slit plane of $0.64 \mathrm{eV} / \mathrm{mm}$. The resolution of the monochromator was $\Delta E=7 \mathrm{meV}$, which corresponds to an energy bandwidth

(a)
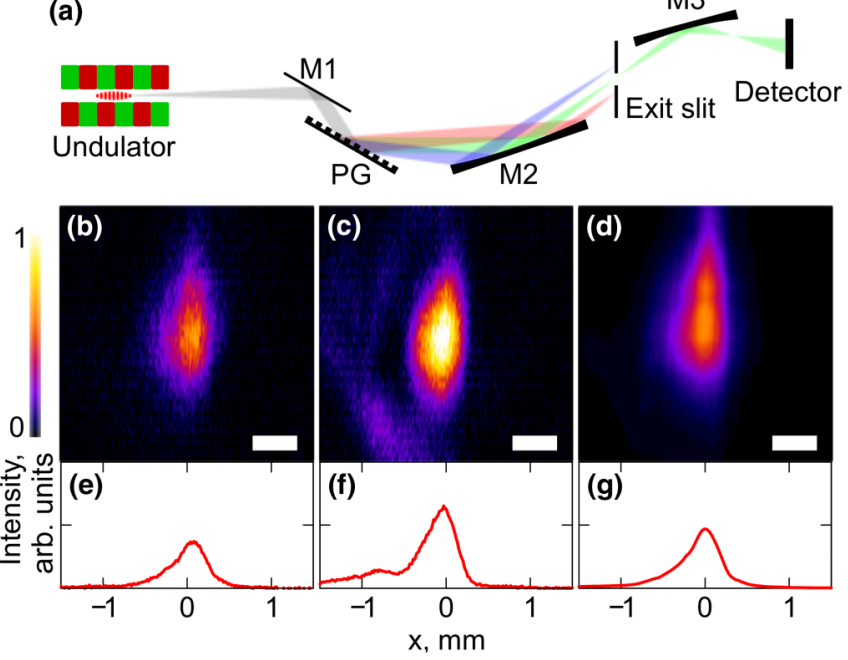

FIG. 1 (color online). (a) Scheme of the experiment. FEL radiation is generated in the undulator and is transmitted through the beam line including one collimating (M1) and two focusing (M2, M3) mirrors, a plane grating (PG), and an exit slit. Intensity profiles of individual femtosecond pulses are measured at the detector. (b),(c) Typical single pulse intensity profiles and (d) an average over $2 \times 10^{4}$ pulses for a bandwidth of $\Delta E / E=0.8 \times$ $10^{-4}$. (e) $-(\mathrm{g})$ Projections of the pulse intensities along the vertical direction. The scale bar is $0.5 \mathrm{~mm}$ long. of $\Delta E / E=3.1 \times 10^{-5}$. Exit slits of 30,60,150,300, and $500 \mu \mathrm{m}$ in size were used to select the spectral width. These values correspond to energy bandwidths $\Delta E / E$ of $0.8 \times$ $10^{-4}, 1.7 \times 10^{-4}, 4.0 \times 10^{-4}, 0.8 \times 10^{-3}$, and $1.4 \times 10^{-3}$.

In contrast to the original HBT experiment $[1,2]$ with two separated detectors, we utilize a pixel detector for correlation measurements. It allows us to determine second- and higher-order correlation functions at all separation points simultaneously. An in-vacuum CCD (Andor Ikon, $2048 \times$ 2048 pixels, each $13.5 \mu \mathrm{m} \times 13.5 \mu \mathrm{m}$ in size) was positioned at a distance of $3.3 \mathrm{~m}$ behind the focus of the beam line. The detector was operated at a repetition rate of $10 \mathrm{~Hz}$ with a region of interest $2047(\mathrm{H}) \times 460(\mathrm{~V})$ pixels and binning by five pixels in the vertical direction. A silicon nitride film $14 \mathrm{~mm} \times 14 \mathrm{~mm}$ in size and $1 \mu \mathrm{m}$ thick was positioned approximately $30 \mathrm{~cm}$ upstream from the camera to attenuate the beam. The transmission of the film at this energy was $4 \times 10^{-6}$. About $2 \times 10^{4}$ intensity profiles were recorded for each monochromator setting [24]. Typical single pulse and averaged intensities are shown in Figs. 1(b)-1(d). Shot-to-shot fluctuations in these intensity profiles, as a consequence of the SASE process are clearly visible. The intensity profiles were averaged along the vertical direction, which is the dispersive direction of the monochromator [see Figs. 1(e)-1(g)]. The intensity correlation analysis was performed in the horizontal direction.

The normalized second-order correlation function $g^{(2)}\left(x_{1}, x_{2}\right)$ for a narrow bandwidth of $0.8 \times 10^{-4}$ is shown in Figs. 2(a) and 2(c). Remarkably, it reaches the maximum value of two at small separations [see Fig. 2(c)], which indicates that the contrast $\zeta_{2}\left(D_{\omega}\right)$ at that monochromator setting is close to one. This contrast is significantly higher than at synchrotron sources [30-32], where it did not exceed 0.3 . This $100 \%$ contrast indicates that at these conditions there is no degradation of the averaged coherence due to optics imperfection. A Gaussian fit, $1+\exp \left(\left(-\Delta x^{2}\right) / l_{c}^{2}\right)$, to the second-order correlation function $g^{(2)}(\Delta x)$ as a function of the separation $\Delta x=x_{2}-x_{1}$ around the center of the beam [see Fig. 2(c)] provided a transverse coherence length of $l_{c}=0.93 \pm 0.04 \mathrm{~mm}$. This value is substantially larger than the measured beam size in the horizontal direction [0.45 mm (FWHM)], indicating a high coherence of the beam. We quantified it by evaluating the degree of spatial coherence $\zeta_{S}$ defined as $[27,33] \zeta_{S}=\int\left|W\left(x_{1}, x_{2}\right)\right|^{2} d x_{1} d x_{2} /$ $\left(\int S(x) d x\right)^{2}$ and obtained $\zeta_{S}=0.78 \pm 0.01$, which is in a good agreement with the Young's double pinhole measurements at FLASH [21]. Importantly, we can estimate the degeneracy parameter [15], which is the number of photons in a single mode. Our estimates, obtained by integrating the total flux on the detector at the narrow bandwidth, yield a value of $10^{9}$ comparable to Ref. [21], which is significantly higher than at any synchrotron sources.

The second-order correlation function $g^{(2)}\left(x_{1}, x_{2}\right)$ for a larger bandwidth of $1.4 \times 10^{-3}$ is shown in Figs. 2(b) and 2(d). This larger bandwidth is equivalent to a shorter 

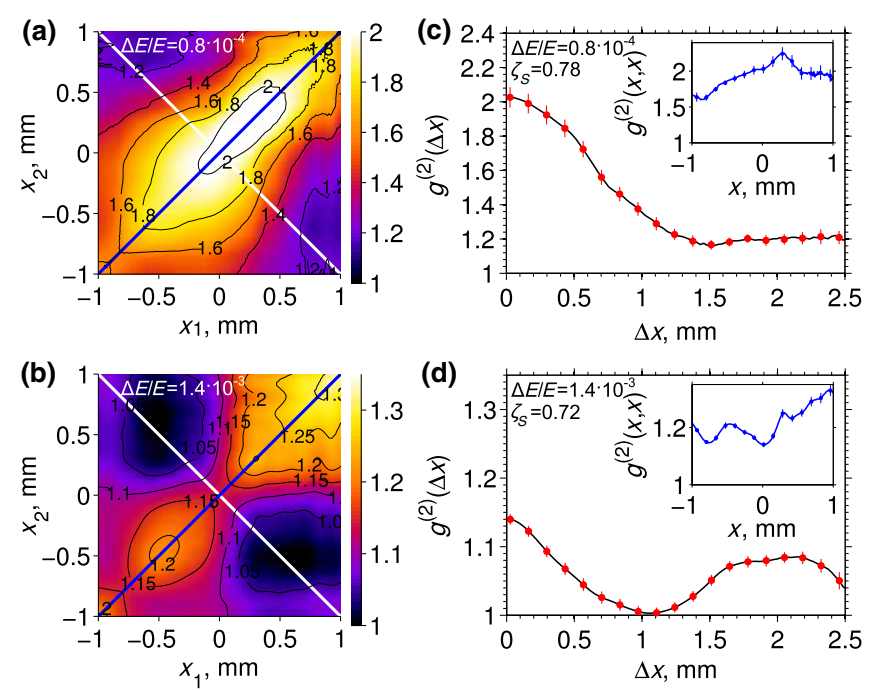

FIG. 2 (color online). Intensity correlation analysis. (a),(b) Intensity correlation function $g^{(2)}\left(x_{1}, x_{2}\right)$ for a bandwidth of $0.8 \times 10^{-4}$ (a) and $1.4 \times 10^{-3}$ (b). (c),(d) Intensity correlation function $g^{(2)}(\Delta x)$ taken along the white line in (a),(b) around the center of the beam. Insets in (c),(d) show intensity fluctuations $g^{(2)}(x, x)=\left\langle I^{2}(x)\right\rangle /\langle I(x)\rangle^{2}$ taken along the blue line in (a),(b). The error bars in (c),(d) are obtained by statistical analysis of 20 individual subensembles $\left(10^{3}\right.$ shots each) from the whole set of $2 \times 10^{4}$ shots. The number of points displayed in this figure with error bars is reduced for better visibility.

coherence time, and the contrast of $g^{(2)}\left(x_{1}, x_{2}\right)$ is reduced as expected from Eq. (2). Unexpectedly, at these conditions we observed an oscillatory shape of the correlation function $g^{(2)}(\Delta x)$ [see Fig. 2(d)]. This may originate from the contribution of two independent sources in the lasing conditions of FLASH [34]. It could also be a more general feature of the nonlinear coupling of the electrons and emitted SASE radiation. To disentangle this question, further experimental and simulation studies of the FEL radiation at different lasing conditions will be required. We want to remark that such fine features of FEL lasing would be difficult to observe by other means. For example, our averaged spectral measurements [see Fig. 3(b)] do not show any notable features.

Results of our experiments also indicate that the statistical properties of the FEL beam are not spatially uniform. This is well visualized by the inspection of the beam fluctuations $g^{(2)}(x, x)=\left\langle I^{2}(x)\right\rangle /\langle I(x)\rangle^{2}$ along the beam profile [see insets in Figs. 2(c) and 2(d)]. They vary significantly both for small and large bandwidths.

We also analyzed the contrast in the center of the beam $\zeta_{2}\left(D_{\omega}\right)=g^{(2)}(0)-1$ as a function of the coherence time $\tau_{c}$ [see Fig. 3(a)]. It has a linear dependence as a function of $\tau_{c}$ at a large bandwidth and reaches saturation at a small bandwidth, as described by Eq. (2) [24]. A theoretical fit to the contrast values yields an average pulse duration of $47 \pm 21 \mathrm{fs}$ (FWHM).

To get an independent estimate of the pulse duration, we measured the second-order correlation function
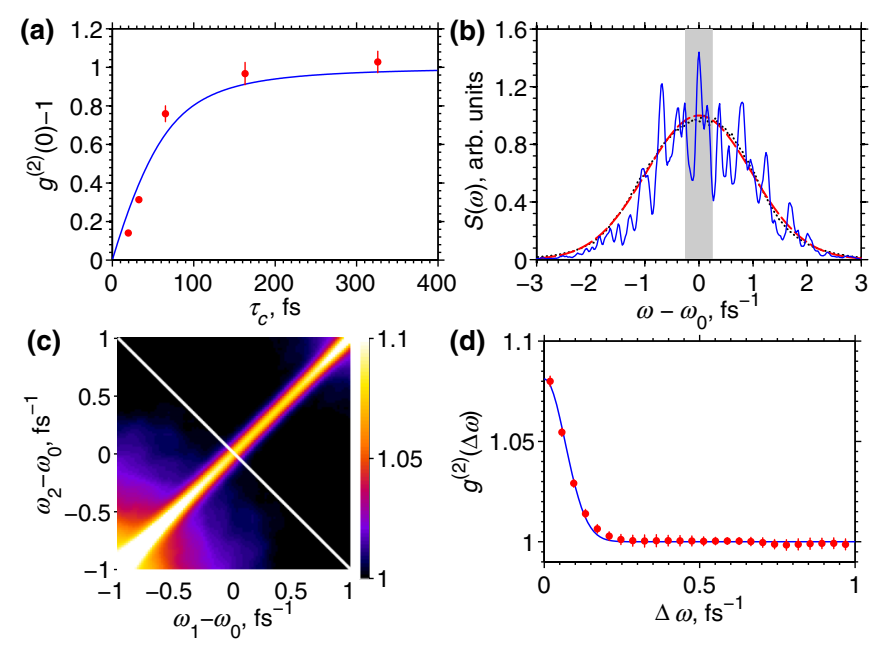

FIG. 3 (color online). Statistical properties of FEL light as a function of the bandwidth. (a) Contrast (points) $\zeta_{2}\left(D_{\omega}\right)=$ $g^{(2)}(0)-1$ as a function the coherence time $\tau_{c}$. The error bars are same as in Figs. 2(c) and 2(d). Theoretical fit (solid line) corresponds to a pulse duration of $T=47 \pm 21 \mathrm{fs}$. (b) Measured single pulse (blue solid line) and average (black dotted line) spectra. Gaussian fit (red dashed line) gives an FEL bandwidth of $\Delta E / E=6.7 \times 10^{-3} \mathrm{FWHM}$. The width of the largest exit slit used in the experiment and corresponding to the bandwidth $\Delta E / E=1.4 \times 10^{-3}$ is shown by the gray region. (c) Intensity correlation function $g^{(2)}\left(\omega_{1}, \omega_{2}\right)$ in the spectral domain. (d) Intensity correlation function $g^{(2)}(\Delta \omega)$ (red circles) taken along the white line in (c). Gaussian fit (blue line) gives an average pulse duration of $27 \mathrm{fs}$.

$g^{(2)}\left(\omega_{1}, \omega_{2}\right)$ in the spectral domain as a function of two frequencies [see Fig. 3(c)] [36,37]. About $1.5 \times 10^{4}$ single shot spectra were recorded after the intensity correlation measurements with a detector at the position of the exit slit of the monochromator. This detector is comprised of a scintillating screen (YAG:Ce 0.2\%) and an intensified CCD (Andor iStar, DH740), equipped with a lens. The effective pixel size of the detector in the exit slit plane was $19.4 \mu \mathrm{m}$ with the point spread function estimated to be about two pixels (FWHM). The detector was operated at a repetition rate of $10 \mathrm{~Hz}$. Line profiles, obtained from the central part of the beam in the horizontal direction corresponding to about $10 \%$ of the beam FWHM were analyzed [24]. A Gaussian fit $1+\exp \left(-\Delta \omega^{2} T^{2}\right)$ was used to determine the pulse duration $T$ from the measurements of the second-order correlation function $g^{(2)}(\Delta \omega)$ [see Fig. 3(d)]. According to this analysis we obtained an averaged pulse duration of $27 \mathrm{fs}$ (FWHM), which lies in the uncertainty range of the previous measurements. In general, our estimates of the pulse duration can be shorter than the intrinsic pulse duration in time domain due to a possible frequency chirp of FLASH pulses [37-39].

To get an insight into the photon statistics of FEL pulses we studied intensity correlation functions of order higher than two. Higher-order correlation functions $g^{(3)}\left(x_{1}, x_{2}, x_{3}\right)$ (with $x_{3}=0$ ) and $g^{(4)}\left(x_{1}, x_{2}, x_{3}, x_{4}\right)$ (with $x_{3}=x_{1} / 2$ and 

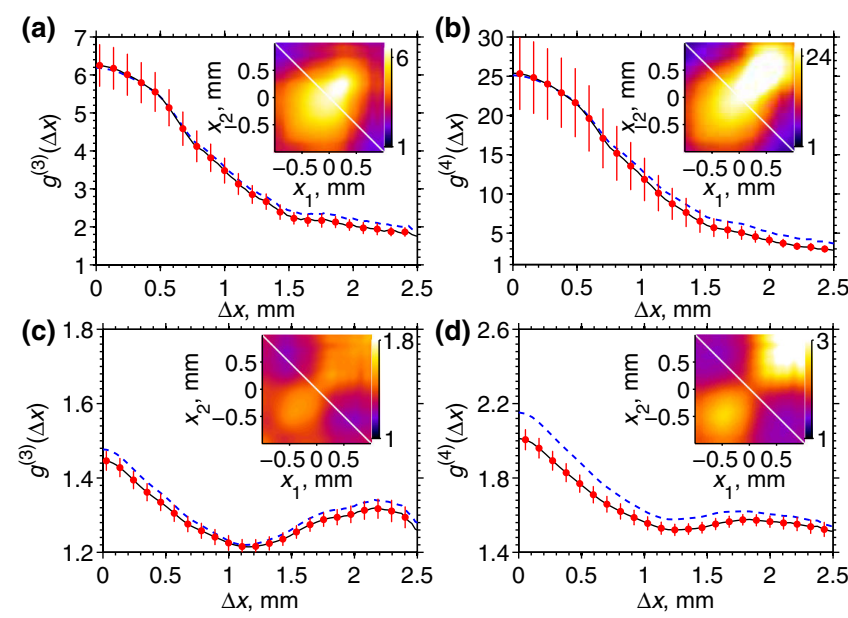

FIG. 4 (color online). Higher order correlation functions for a bandwidth of $0.8 \times 10^{-4}$ (a),(b) and $1.4 \times 10^{-3}$ (c),(d). (a),(c) Third-order correlation function $g^{(3)}\left(x_{1}, x_{2}\right)$ (shown in the insets) and $g^{(3)}(\Delta x)$ (black solid line) taken along the white line in the insets. (b,d) Fourth-order correlation function $g^{(4)}\left(x_{1}, x_{2}\right)$ (shown in the insets) and $g^{(4)}(\Delta x)$ (black solid line) taken along the white line in the insets. The same functions obtained under assumption of the Gaussian statistics are shown as blue dashed lines. The number of points displayed in this figure with error bars is reduced for better visibility. Error bars are evaluated in the same way as in Fig. 2.

$\left.x_{4}=x_{2} / 2\right)$ are presented in the insets of Fig. 4 for two different bandwidths as functions of $x_{1}$ and $x_{2}$. In Fig. 4 the same quantities are presented as a function of $\Delta x$ around the center of the beam with $x_{1}=-\Delta x / 2, x_{2}=\Delta x / 2$. The higher-order correlation functions were also calculated under the assumption of Gaussian statistics [24] from the measured second-order correlation function $g^{(2)}\left(x_{1}, x_{2}\right)$ (see Fig. 4). The excellent agreement between these two curves confirms the chaotic nature of the FLASH source at these operation conditions. In particular, at a small bandwidth we observed a factorial behavior of higher order correlation functions at zero point separation $g^{(n)}(\mathbf{r}, \mathbf{r}, \ldots, \mathbf{r}) \approx n !$ [see Figs. 4(a) and 4(b)], which is typical for Gaussian statistics. At a wider bandwidth the maximum value is substantially reduced compared to a narrow bandwidth case [see Figs. 4(c) and 4(d)].

In summary, we have presented high-order intensity correlation measurements at FLASH which provide a simple, robust, and versatile tool for monitoring basic beam properties of FELs including the degree of spatial coherence, average pulse duration, and details of the photon statistics. We obtained an averaged pulse duration less than $50 \mathrm{fs}$, degree of transverse coherence of about $80 \%$, and degeneracy parameter of more than $10^{9}$. Such values are similar to laser sources and were never observed at these wavelengths at conventional synchrotron radiation sources. However, our measurements of the higher-order correlation functions indicate that present FEL sources based on the SASE principle are essentially chaotic sources obeying Gaussian statistics.
The measurements presented in this work were performed at XUV photon energies that can be conveniently reached at FLASH. We anticipate that there are no principal limitations to extend our approach to every existing or planned FEL source, including hard x-ray FELs. This is in contrast to Young's double pinhole experiments, which are limited to the soft $\mathrm{x}$-ray energy region due to high penetration depth of hard $\mathrm{x}$ rays and difficulties of manufacturing thick opaque apertures.

An interesting further application of the methods developed in this work would be the study of seeded FEL sources [40-42]. An intriguing question is whether seeded FELs are fully coherent sources in all orders according to Glauber [3] and, in this way, are equivalent to conventional single mode lasers, or if they obey Gaussian statistics like SASE FELs. We could also foresee that intensity correlation analysis might be applied to study the dynamics of ultrafast processes at FELs. For example, the intensity correlation analysis of the Coulomb explosion in single molecule imaging experiments [43] could provide detailed information about the disintegration of these molecules on a femtosecond time scale. The exploding molecule can be considered as a secondary source and its size at different instants of time can be studied using the intensity correlation method.

We acknowledge fruitful discussions with E. Saldin, E. Schneidmiller, and M. Altarelli, the development of cross correlation analysis in frequency domain by S. Serkez, and careful reading of the manuscript by R. Röhlsberger. Part of this work was supported by BMBF Grant No. 5K10CHG "Coherent Diffraction Imaging and Scattering of Ultrashort Coherent Pulses with Matter" in the framework of the German-Russian collaboration "Development and Use of Accelerator-Based Photon Sources" and the Virtual Institute VH-VI-403 of the Helmholtz Association. Funding within the BMBF priority Grant No. FSP-301 FLASH and through the DFG Grant No. GRK1355 "Physics with new advanced coherent light sources" is gratefully acknowledged. We also are greatly indebted to the scientific and technical team at FLASH, in particular, the machine operators and run coordinators, being the foundation of the successful operation and delivery of the SASE-FEL beam.

\footnotetext{
*Present address: Helmholtz-Zentrum Berlin für Materialien und Energie GmbH, Albert-Einstein-Str 15, D-12489 Berlin, Germany.

${ }^{\dagger}$ Present address: Institut für Regenerative EnergieSysteme, Zur Schwedenschanze 15, D-18435 Stralsund, Germany.

*Present address: Center for Free-Electron Lasers, Notkestrasse 85, D-22607 Hamburg, Germany.

${ }^{\S}$ Corresponding author.

Wilfried.Wurth@desy.de
} 
"Corresponding author. Ivan.Vartaniants@desy.de

[1] R. Hanbury Brown and R. Q. Twiss, Nature (London) 177, 27 (1956).

[2] R. H. Brown and R. Q. Twiss, Nature (London) 178, 1046 (1956).

[3] R. J. Glauber, Phys. Rev. 130, 2529 (1963).

[4] F. T. Arecchi, E. Gatti, and A. Sona, Phys. Lett. 20, 27 (1966).

[5] M. Aßmann, F. Veit, M. Bayer, M. van der Poel, and J. M. Hvam, Science 325, 297 (2009).

[6] S. S. Hodgman, R. G. Dall, A. G. Manning, K.G.H. Baldwin, and A. G. Truscott, Science 331, 1046 (2011).

[7] A. Perrin, R. Bücker, S. Manz, T. Betz, C. Koller, T. Plisson, T. Schumm, and J. Schmiedmayer, Nat. Phys. 8, 195 (2012).

[8] W. Ackermann et al., Nat. Photonics 1, 336 (2007).

[9] P. Emma et al., Nat. Photonics 4, 641 (2010).

[10] T. Ishikawa et al., Nat. Photonics 6, 540 (2012).

[11] The European X-Ray Free-Electron Laser Technical Design Report, DESY 2006-097, 2006, edited by M. Altarelli et al., http://xfel.desy.de/technical_information/ $\mathrm{tdr} / \mathrm{tdr} /$.

[12] H. Chapman et al., Nature (London) 470, 73 (2011).

[13] K. J. Gaffney and H.N. Chapman, Science 316, 1444 (2007).

[14] M. Seibert et al., Nature (London) 470, 78 (2011).

[15] L. Mandel and E. Wolf, Optical Coherence and Quantum Optics (Cambridge University Press, Cambridge, England, 1995).

[16] J.W. Goodman, Statistical Optics (Wiley, New York, 1985).

[17] A. Singer, I. Vartanyants, M. Kuhlmann, S. Duesterer, R. Treusch, and J. Feldhaus, Phys. Rev. Lett. 101, 254801 (2008).

[18] R. Mitzner et al., Opt. Express 16, 19909 (2008).

[19] W. F. Schlotter, F. Sorgenfrei, T. Beeck, M. Beye, S. Gieschen, H. Meyer, M. Nagasono, A. Föhlisch, and W. Wurth, Opt. Lett. 35, 372 (2010).

[20] I. A. Vartanyants et al., Phys. Rev. Lett. 107, 144801 (2011).

[21] A. Singer et al., Opt. Express 20, 17480 (2012).

[22] C. Gutt et al., Phys. Rev. Lett. 108, 024801 (2012).
[23] H. Paul, Rev. Mod. Phys. 58, 209 (1986).

[24] See Supplemental Material at http://link.aps.org/ supplemental/10.1103/PhysRevLett.111.034802 for the expressions of the higher-order correlation functions in the frame of Gaussian statistics, the determination of the pulse duration from the intensity correlation measurements, and results of the measurements from all monochromator settings.

[25] E. Ikonen, Phys. Rev. Lett. 68, 2759 (1992).

[26] E. L. Saldin, E. A. Schneidmiller, and M. V. Yurkov, The Physics of Free Electron Lasers (Springer-Verlag, Berlin, 2000).

[27] E. L. Saldin, E. A. Schneidmiller, and M. V. Yurkov, Opt. Commun. 281, 1179 (2008).

[28] M. Martins, M. Wellhöfer, J. T. Hoeft, W. Wurth, J. Feldhaus, and R. Follath, Rev. Sci. Instrum. 77, 115108 (2006).

[29] N. Gerasimova, S. Dziarzhytski, and J. Feldhaus, J. Mod. Opt. 58, 1480 (2011).

[30] E. Gluskin, E. E. Alp, I. McNulty, W. Sturhahn, and J. Sutter, J. Synchrotron Radiat. 6, 1065 (1999).

[31] M. Yabashi, K. Tamasaku, and T. Ishikawa, Phys. Rev. Lett. 87, 140801 (2001).

[32] M. Yabashi, K. Tamasaku, and T. Ishikawa, Phys. Rev. Lett. 88, 244801 (2002).

[33] I. A. Vartanyants and A. Singer, New J. Phys. 12, 035004 (2010).

[34] See, for example, general theoretical analysis of HBT experiment in the presence of two independent sources developed in Ref. [35].

[35] G. Baym, Acta Phys. Pol. B 29, 1839 (1998).

[36] A. A. Lutman, Y. Ding, Y. Feng, Z. Huang, M. Messerschmidt, J. Wu, and J. Krzywinski, Phys. Rev. ST Accel. Beams 15, 030705 (2012).

[37] Y. Inubushi et al., Phys. Rev. Lett. 109, 144801 (2012).

[38] U. Frühling et al., Nat. Photonics 3, 523 (2009).

[39] I. Grguras et al., Nat. Photonics 6, 852 (2012).

[40] G. Geloni, V. Kocharyan, and E. Saldin, J. Mod. Opt. 58, 1391 (2011)

[41] J. Amann et al., Nat. Photonics 6, 693 (2012).

[42] E. Allaria et al., Nat. Photonics 6, 699 (2012).

[43] R. Neutze, R. Wouts, D. van der Spoel, E. Weckert, and J. Hajdu, Nature (London) 406, 752 (2000). 